\title{
2-S21-2
}

\section{Development of liver organoid culture from NASH mouse model and the application to drug discovery}

\section{Tatsuya Usui}

\section{Tokyo University of Agriculture and Technology}

In recent years, the number of non-alcoholic steatohepatitis (NASH) patients, who develop fatty liver in an alcoholindependent manner and progress to cirrhosis and liver cancer in the future, has been increasing, and there are more than approximately 10 million patients and their preps in Japan. The three-dimensional organoid culture method has been attracting attention because it enables us to culture epithelial cells and epithelial stem cells in a threedimensional manner for a long period of time by culturing them in a special culture medium containing factors that enhance the stem cell nature and maintain the original characteristics of the tissue, such as heterogeneity and polarity. Recently, we have developed a new three-dimensional organoid culture model for recapitulating liver fibrosis by focusing on the liver tissue from a mouse model of NASH.

Six-week-old C57/BL mice were fed a NASH-inducing diet for 4, 8, and 12 weeks, and different stages of NASH progression (NASH A, NASH B, and NASH C) were cultured in NASH model mice. In the liver tissues of the NASH model mice at each stage, fibrosis of the liver, deposition of fatty droplets, and elevated markers of liver damage were observed with prolongation of the feeding period, while epithelial and mesenchymal transition (EMT)-like epithelial tissue structures were observed in the NASH C organoids, with accumulation of collagen and activated star cell markers, such as -SMA expression was found to be up-regulated in organoids. Furthermore, we identified several genes that were specifically up-regulated in organoids of each pathological stage and genes that were highly expressed in organoids of all stages (JP 2019-230780). These results indicate that liver-derived organoids from mouse models of NASH at different stages of progression recapitulate the fibrotic pathology of NASH on culture dishes. In addition, our results suggest that genes up-regulated in NASH organoids may become novel biomarkers in the future 